Results Eight of the 27 patients had one abnormal test, six had two and in five, all three tests were abnormal. In fifteen of the 27 patients with a normal GES (56\%), eight had normal GEBT and GUS studies. Of the remaining seven patients, four had a normal GEBT and an abnormal GUS, two had normal GUS with an abnormal GEBT, and in one, both the GEBT and GUS were abnormal. GES was delayed in ten of the 27 patients (37\%). In four of these, both GEBT and GUS were abnormal, three had delayed gastric emptying on GEBT with a normal GUS, two had delayed gastric emptying on GUS with normal GEBT, and in one patient, both GUS and GEBT were normal. GES was abnormally rapid in two patients (7\%). In one patient, both GEBT and GUS indicated rapid gastric emptying and in the other, GUS revealed rapid gastric emptying with a normal GEBT. Assuming GES as the gold standard for diagnosing abnormal gastric emptying, GUS has a sensitivity and specificity for detecting a motor disorder of $66 \%$ and GEBT has a sensitivity of $66 \%$ and a specificity of $80 \%$.

Conclusion In this group of FD patients, $70 \%$ had at least one abnormal test of gastric motor function. Whilst GES is regarded as the gold standard test, in seven patients with normal GES, the GEBT, GUS, or both, were abnormal. This discrepancy might reflect the day-to-day variability of gastric motor function testing or that each investigation measures a different component of gastric motor physiology. We conclude that in FD, adding GEBT and GUS to GES substantially increases the positive diagnostic yield and the heterogeneous patterns might indicate a variety of FD subtypes.

Disclosure of Interest None Declared.

\section{PTU-147 VALIDATION OF UPDATED BARRETT'S OESOPHAGUS GUIDELINE RECOMMENDATIONS: COMPLICATIONS AND OUTCOMES OF AN 'ADEQUATE' CASE VOLUME AND THE FEASIBILITY OF SAME DAY DISCHARGE}

E Taylor*, S Din, J Campbell, A Hopper. Gastroenterology, Sheffield Teaching Hospitals, Sheffield, UK

\subsection{6/gutjnl-2014-307263.22}

Introduction Recent advances in the management of high grade dysplasia and early cancer in Barrett's oesophagus (BO) have led to updated guidelines recommending endoscopic resection (ER) as a first line option in selected cases. The complexity and complications of ER have prompted guidelines recommending at least 15 procedures annually per endoscopist to minimise complications. The reported complications of oesophageal perforation $(<0.9 \%)$ and delayed bleeding $(2-22 \%)$ may prompt routine overnight observation following resection. Our aim was to report complications and outcomes in a unit performing just above the recommended annual numbers of ER for BO.

Methods All patients undergoing ER for BO over 3 years were identified. All resections were performed with a mutiband ligation technique. Number of resections performed, size of resected specimens and stage of dysplasia/cancer in specimens were recorded. Complications of delayed bleeding, perforation, or dysphagia requiring dilation were also recorded.

Results In 3 years, 108 endoscopic resections were performed in 46 procedures (median resections per procedure $=2$; range 1-6). 3 patients underwent 2 separate procedures. Resected specimens ranged in size from 3-17 mm (mean 10mm, SD 2.77). Final histological diagnosis per procedure was: no dysplasia $=6$, low grade dysplasia $=3$, high grade dysplasia $=8$, invasive cancer- $\mathrm{T} 1 \mathrm{a}=9 \mathrm{~T} 1 \mathrm{~b}$ $=13, \mathrm{~T} 2=4.2$ ER specimens were not retrieved, 1 showed granular cell carcinoma. Immediate complications included 1 perforation (2.3\%. 95\% CI:0-13\%) with successful closure at endoscopy. 2 procedures were abandoned due to immediate bleeding (4.6\% 95\% CI 4.2 to $16.0 \%$ ) which was successfully treated at the time. Delayed bleeding occurred in 2 patients, (4.6\% 95\% CI 4.2 to $16.0 \%)$ requiring emergency OGD at $8 \mathrm{~h}$ and 11 days post procedure. The second required endotherapy and readmission for 7 nights. 7 patients developed post-ER dysphagia (15.9\% 95\% CI 7.6 to 29.7 ) requiring oesophageal dilation (median procedures $=2$, range $1-5$ ). There was no significant difference in the number of resections in patients who had perforation (1 ER) or bleeding (median 1 range $1-3) \mathrm{p}=0.56$. Patients who developed symptomatic strictures had a significantly higher number of resections (median 4 range 1-6) p $<0.0001$.

Conclusion Complication rates of Barrett's ER procedures in a unit performing an adequate number are comparable to published outcomes from high volume centres. Delayed bleeding is rare, occurring up to 7 days post procedure and is not more common within the first $24 \mathrm{~h}$. Therefore, if no immediate complications occur, same day discharge is appropriate.

Disclosure of Interest None Declared.

\section{PTU-148 HEALTHCARE COSTS AND QUALITY OF LIFE ASSOCIATED WITH ACUTE UPPER GASTROINTESTINAL BLEEDING IN THE UK}

${ }^{1}$ E Stokes ${ }^{*},{ }^{1} \mathrm{H}$ Campbell, ${ }^{1} \mathrm{D}$ Bargo, ${ }^{2} \mathrm{M}$ Murphy, ${ }^{3} \mathrm{R}$ Logan, ${ }^{4} \mathrm{~V}$ Jairath on behalf of TRIGGER Investigators. ${ }^{1}$ Health Economics Research Centre, Nuffield Department of Population Health, University of Oxford, UK; ${ }^{2}$ Transfusion Medicine, NHS Blood and Transplant, Oxford, UK; ${ }^{3}$ University of Nottingham, Nottingham, UK; ${ }^{4}$ Translational Gastroenterology Unit, Nuffield Department of Medicine, Oxford, UK

\subsection{6/gutjpl-2014-307263.222}

Introduction Acute upper gastrointestinal bleeding (AUGIB) accounts for over 70,000 hospital admissions in the UK annually. Its incidence is likely to rise due to an ageing population and increasing burden of liver disease. Data on the healthcare costs and health-related quality of life (HRQoL) associated with this condition are sparse.

Methods The TRIGGER trial is a cluster randomised feasibility trial evaluating restrictive versus liberal red cell transfusion for patients with AUGIB. The study collected data on resource use, costs and outcomes during hospitalisation and up to day 28 to explore the feasibility of gathering inputs required for a costeffectiveness analysis. Resource use data were collected during the inpatient episode on the use of laboratory tests, medications, blood components, endoscopy and endoscopic therapy, clinical events including ischaemic/thromboembolic events and length of hospital stay (LOS) by ward type. Data were also collected on primary and secondary care resource use, as well as informal care/days off work, post-discharge to day 28. Resource use for each patient was multiplied by national unit costs to generate an estimate of the costs of AUGIB to 28 days. HRQoL was measured on a scale anchored at 0 (death) and 1 (full health), using the EuroQol EQ-5D-3L questionnaire at day 28.

Results 936 patients were enrolled into TRIGGER between August 2012 and March 2013 in 6 UK hospitals. Preliminary analyses show that the mean (standard error (SE)) cost of the inpatient episode was $£ 1,914$ ( $£ 78)$ per patient. LOS was a key cost driver; mean LOS was 5.4 days with an associated cost of $£ 1431$. Additional cost drivers included: (1) red cell transfusion, with a mean of 1.6 units transfused per patient at a cost of $£ 197$; (2) endoscopy, with mean of 0.8 endoscopies per patient 